\section{Nasopharyngeal Rhabdomyosarcoma in a Child : A Case Report}

Ilssam Lalya,

Hassan Sifat, Khalid Hadadi Mansouri Hamid
Rhabdomyosarcoma (RMS) is a highly malignant neoplasm that arises from embryonic mesenchyme [1]. It is the most common soft tissue tumour in children, with the head and neck region accounting for $35-40 \%$ of cases [2]. Para meningeal (PM) rhabdomyosarcomas are tumors that arise from sites adjacent to the meninges, including the nasopharynx and nasal cavity, middle ear and mastoid, para nasal sinuses, pterygopalatine and infratemporal fossa. They have a propensity for central nervous system extension and poorer survival than the others rhabdomyosarcoma. The presentation in the nasopharynx is very rare only few cases have been reported in the literature [3]. External beam radiotherapy is an integral component of treatment for nasopharyngeal RMSs [2].We report the case of an Arabian 6 year old boy, presented to our institution for a 6 months history of nasal obstruction and right cervical lymphadenopathy. Nasopharyngeal endoscopy with biopsy showed a mass involving the entire nasopharynx. Histologic analysis revealed embryonic rhabdomyosarcoma. Magnetic resonance imaging showed an organic process of the nasopharynx invading parapharyngeal spaces, the pterygoide processes, prevertebral and retrostyloid spaces without intracranial extension (Figure 1 and Figure 2). Work-up including chest and abdomen CT scan and bone scintigraphy was normal. The patient received 8 cycles of VAC (Vincristine, Adriamycin, Cyclophosphamide) followed by radiation therapy at the dose of 50, 4 Gy. The first MRI of assessment showed a complete response.

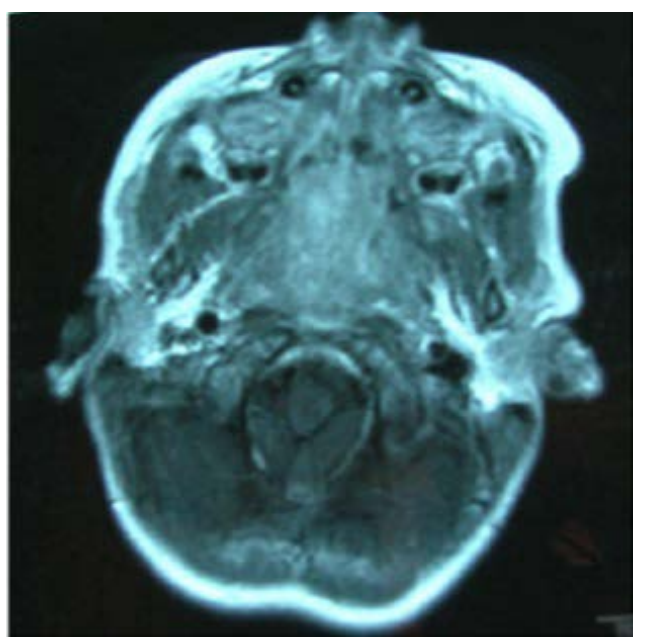

Figure 1 Axial MRI showing the nasopharyngeal process.

\section{Department of Radiation Therapy, Military Teaching Hospital Mohammed V, Rabat, Morocco}

Corresponding author: Issam Lalya

झ issamlalya@yahoo.fr

Department of Radiation Therapy, Military Teaching Hospital Mohammed V, Rabat, Morocco

Tel: 00212661572770

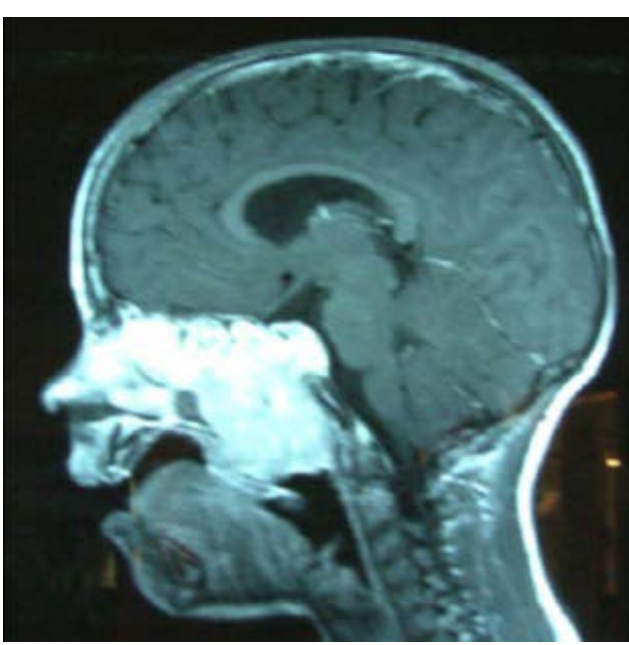

Figure 2 Sagittal MRI showing no intracranial extension. 


\section{References}

1 Horn RC, Enterhne HT (1958) Rhabdomyosarcorna, a cllncopathologlcal study and classification. Cancer 11: 181.

2 Healy JN, Borg MF (2010) Paediatric nasopharyngeal rhabdomyosarcoma: A case series and literature review. J Med Imaging Radiat Oncol 54: 388-394.

3 Desarda KK, Gill SJ, Bora MP (2000) Nasopharyngeal rhabdomyosarcoma. Indian Journal of Otolaryngology and Head and Neck Surgery 52: 173-174. 